\title{
Effects of an 11-nm DMSA-coated iron nanoparticle on the gene expression profile of two human cell lines, THP-1 and HepG2
}

Ling Zhang ${ }^{1,2}$, Xin Wang ${ }^{1}$, Jinglu Zou' ${ }^{1}$ Yingxun Liu ${ }^{1}$ and Jinke Wang ${ }^{1 *}$

\begin{abstract}
Background: Iron nanoparticles (FeNPs) have attracted increasing attention over the past two decades owing to their promising application as biomedical agents. However, to ensure safe application, their potential nanotoxicity should be carefully and thoroughly evaluated. Studies on the effects of FeNPs on cells at the transcriptomic level will be helpful for identifying any potential nanotoxicity of FeNPs and providing valuable mechanistic insights into various FeNPs-induced nanotoxicities.

Results: This study investigated the effects of an 11-nm dimercaptosuccinic acid-coated magnetite nanoparticle on the gene expression profiles of two human cell lines, THP-1 and HepG2. It was found that the expression of hundreds of genes was significantly changed by a 24-h treatment with the nanoparticles at two doses, $50 \mu \mathrm{g} / \mathrm{mL}$ and $100 \mathrm{\mu g} / \mathrm{mL}$, in the two cell types. By identifying the differentially expressed genes and annotating their functions, this study characterized the general and cell-specific effects of the nanoparticles on two cell types at the gene, biological process and pathway levels. At these doses, the overall effects of the nanoparticle on the THP-1 cells were the induction of various responses and repression of protein translation, but in the HepG2 cells, the main effects were the promotion of cell metabolism, growth and mobility. In combination with a previous study, this study also characterized the common genes, biological processes and pathways affected by the nanoparticle in two human and mouse cell lines and identified $l d 3$ as a nanotoxicity biomarker of the nanoparticle.
\end{abstract}

Conclusion: The studied FeNPs exerted significant effects on the gene expression profiles of human cells. These effects were highly dependent on the innate biological functions of cells, i.e., the cell types. However, cells can also show some cell type-independent effects such as repression of Id3 expression. Id3 can be used as a nanotoxicity biomarker for iron nanoparticles.

Keywords: Iron nanoparticle, Gene expression profile, THP-1, HepG2

\section{Background}

Iron nanoparticles (FeNPs) have attracted increasing attention over the past two decades owing to their promising applications as biomedical agents $[1,2]$. FeNPs have been the most intensively studied and commercialized nanomaterial in recent years. Despite their generally good biocompatibility relative to other metal nanomaterials [3], their potential nanotoxicity has been recognized $[4,5]$. For this reason, many studies have investigated the potential nanotoxicity of FeNPs [6]. Some important nanotoxicities

\footnotetext{
* Correspondence: wangjinke@seu.edu.cn

'State Key Laboratory of Bioelectronics, Southeast University, Nanjing 210096, China

Full list of author information is available at the end of the article
}

of FeNPs were thus discovered, including reduction of cell viability $[7,8]$ and induction of cellular inflammation $[9,10]$, mitochondrial injury $[8,11,12]$, apoptosis $[8,13,14]$, reactive oxygen species (ROS) $[8,11,15,16]$, autophagy $[8,11]$, oxidative stress $[14,17,18]$, cell motility impairment [15], and DNA damage $[17,18]$.

In response to any intracellular and extracellular environmental changes, cells can rapidly change their transcriptomic output, i.e., gene expression profile. In this way, cells adapt to the environmental changes for their survival and function. However, excessive environmental changes can damage the normal physiological activities and biological functions of cells. Therefore, evaluation of gene expression profile changes is helpful in identifying 
the potential nanotoxicity of nanomaterials [16,19-21]. Identifying all the genes whose expression is affected by a nanomaterial at the cell or tissue levels can provide valuable clues for identifying any potential toxicity and the relevant molecular mechanism [16,19,20,22]. Moreover, current transcriptomic profiling techniques including GeneChip and RNA-seq allow the analysis of global gene expression [20,23,24]. Therefore, increasing numbers of studies have investigated the nanotoxicity of various nanomaterials at the transcriptome level $[16,20,24]$. Many previously unknown nanotoxicities of nanomaterials were thus uncovered, such as intracellular production of ROS and the resulting cell apoptosis induced by silver, silica and magnetic nanoparticles $[19,20,25]$.

Recent transcriptomic studies have provided valuable mechanistic insights into the various nanotoxicities induced by FeNPs. For example, a transcriptomic analysis found that the transcription of many genes relevant to iron metabolism (Trf, Tfrc, Lcn2, Hfe) and osmosis (Slc5a3, Slc6a12) was significantly changed by FeNPs in mouse RAW264.7 cells [21], indicating that the iron and osmotic homeostasis of the cells was disturbed by FeNPs. The subsequent measurement of the cellular iron content revealed that the internalized FeNPs were degraded in the acidic environment of the lysosomes and thus released iron ions in the cells, which changed the iron and osmotic homeostasis of the cells. In complementary responses, the cells downregulated the expression of the Trf, Tfrc, and Hfe genes to prevent the transfer of extracellular $\mathrm{Fe}^{2+}$ into the cells, upregulated the expression of the $L c n 2$ gene to promote the transfer of intracellular $\mathrm{Fe}^{2+}$ out of the cells, and downregulated the expression of the Slc5a3 gene to inhibit the transfer of extracellular myo-inositol, a very important organic osmolyte, into the cells [21].

Our lab has recently evaluated the effects of a FeNP material deemed to have good biocompatibility, 11-nm magnetite $\left(\mathrm{Fe}_{3} \mathrm{O}_{4}\right)$ FeNPs coated with dimercaptosuccinic acid (DMSA) [9], at the transcriptome level. The potential nanotoxicological effects of these FeNPs at doses of 50 and $100 \mu \mathrm{g} / \mathrm{mL}$ on the gene expression profiles of two mouse cell lines (RAW264.7 and Hepa1-6) were examined [10]. This study characterized the general and cell-specific biological processes affected by the FeNPs in these two cell lines by identifying the differentially expressed genes (DEGs) and annotating their functions, providing new insights into the nanotoxicity of the FeNPs. RAW264.7 cells are a blood cell line belonging to monocyte-macrophage system, whereas Hepa1-6 cells are a liver-derived hepatoma cell line. Generally, the former is mainly involved in immune activity, whereas the latter is responsible for detoxification in the living body. The blood and liver cells encounter the greatest exposure to the nanomaterials in vivo due to the use of intravenous administration and the passive targeting of nanomaterials. Therefore, the two cell lines are suitable for evaluating the nanotoxicity of FeNPs.

The benefit of using mouse cells is that the nanotoxicity observed in vitro can be further evaluated in vivo by administering the nanomaterials to mice [26]. However, the similar in vivo evaluation cannot be performed in humans. Therefore, a feasible strategy is to evaluate the nanotoxicity of a nanomaterial with human cells and their mouse equivalents. If the in vitro nanotoxicity of a nanomaterial is similar in cells of two species, its in vivo nanotoxicity can be evaluated in the mouse to judge its in vivo nanotoxicity in humans. According to this strategy, based on our recent study of the nanotoxicity of a FeNP with two mouse cells [10], this study treated two equivalent human cell lines, human monocytic THP-1 cells and hepatoma HepG2 cells, with the same FeNPs at the same doses (50 and $100 \mu \mathrm{g} / \mathrm{mL}$ ) for the same time $(24 \mathrm{~h})$, and profiled the global gene expression with genechips. This study thus identified hundreds of DEGs in two cell lines. By comparing the DEGs, their annotated functions and the associated pathways, this study evaluated the general and cell-specific effects the FeNPs on two human cell lines. By comparing these results with the previously characterized effects of the same FeNPs on two mouse cell lines, this study defined the common effects of the FeNPs on human and mouse cells. This study also identified a cell-independent nanotoxicity biomarker for the FeNPs. Together, the results of this study provide new insights into the nanotoxicity of the FeNPs and the underlying molecular mechanisms.

\section{Results and discussion}

\section{Characterization of FeNPs and their cellular internalization}

The average hydrodynamic size of the FeNPs was $32 \mathrm{~nm}$ (Figure 1A). Zeta potential measurements showed that the FeNPs were negatively charged in water (Figure 1B). The average size of the FeNPs measured by TEM was $11 \pm 1.24 \mathrm{~nm}$. The FeNPs were monodisperse and of uniform size in water (Figure 1C). Prussian blue staining revealed that the FeNPs were taken up by the cells and more nanoparticles were internalized into the cells at the high dose (Figure 1D). The blue staining of the FeNPs agglomerates was clearer in the HepG2 cells than in the THP-1 cells. The reason for the different appearance is that the former is an adherent cell but the latter is a suspension cell.

\section{Identification of FeNP-responsive genes}

The GeneChip analysis identified 287 and 714 genes as DEGs (i.e., FeNP-responsive genes, FeRGs) in the THP-1 cells treated with $50 \mu \mathrm{g} / \mathrm{mL}$ (low dose) and $100 \mu \mathrm{g} / \mathrm{mL}$ (high dose) of FeNPs, respectively. Under the same 

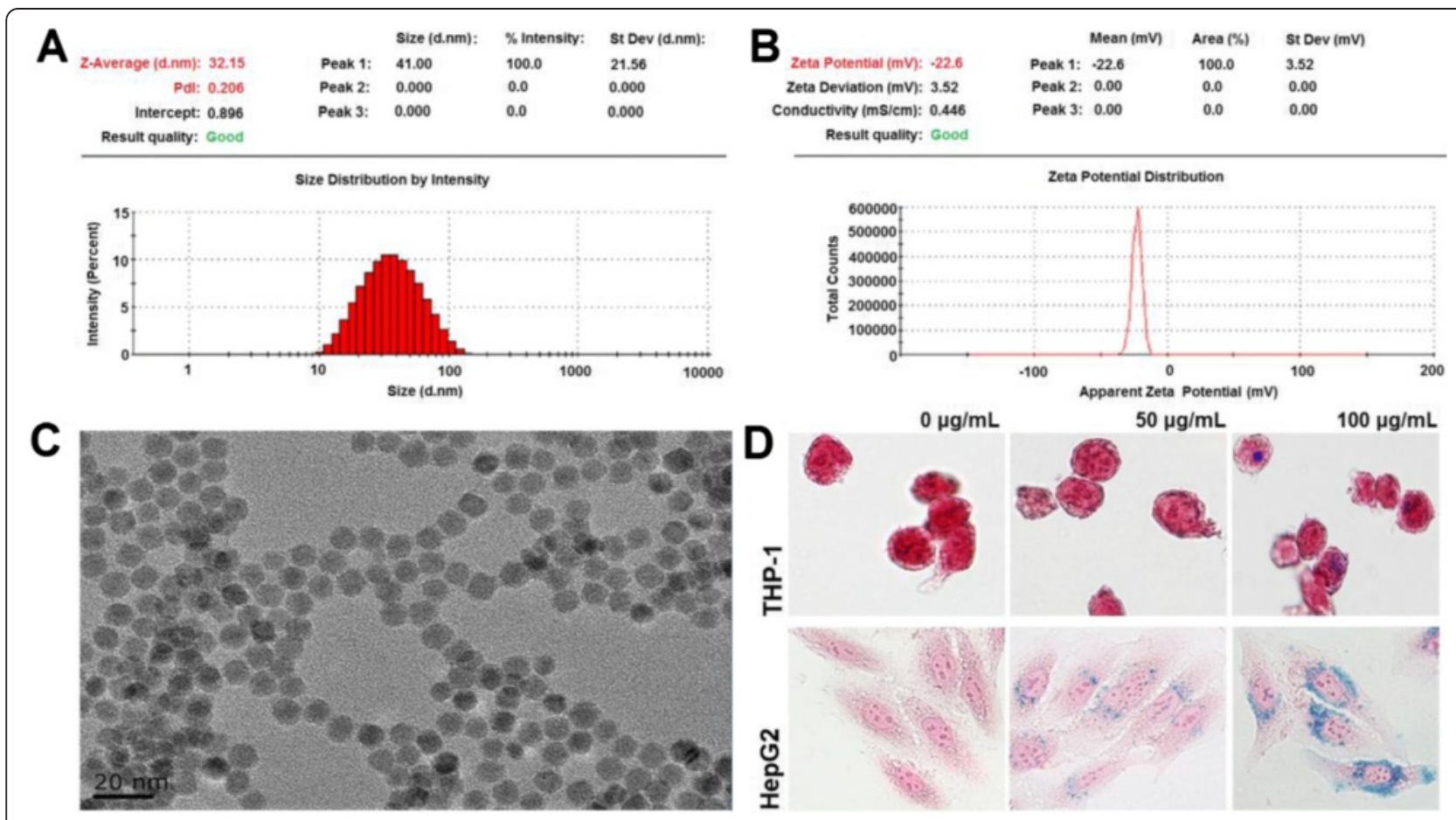

Figure 1 Characterization of the FeNPs and their cellular internalization. A: Hydrodynamic sizes of the FeNPs. B: Zeta potential of the FeNPs. C: TEM observation of FeNPs. D: Prussian blue staining of cells treated with FeNPs at three doses. Magnification, $\times 400$.

conditions, 221 and 265 genes were identified as FeRGs in the HepG2 cells. More genes were regulated by the high-dose FeNPs (hdFeNPs) in the two cell lines, especially in the THP-1 cells. In the THP-1 cells, 229 genes were induced and 58 genes were repressed by the lowdose FeNPs (ldFeNPs), whereas 571 genes were induced and 143 genes were repressed by the hdFeNPs. In the HepG2 cells, 139 genes were induced and 82 genes were repressed by the ldFeNPs, whereas 96 genes were induced and 169 genes were repressed by the hdFeNPs. More genes were induced in the THP-1 cells but repressed in the HepG2 cells. The previous study revealed that more genes were repressed in the RAW264.7 cells but induced in the Hepa1-6 cells [10]. These data indicate that the FeNPs resulted in differential effects on the gene expression patterns of the cells of two species. The expression of some representative genes was confirmed by quantitative PCR (qPCR) analysis (Figure 2).

The genes with highest fold change in transcription revealed the most sensitive responses of the cells to FeNPs at the gene expression level. The top 10 induced and repressed FeRGs in the two cell lines are shown in Figure 3. Clearly, the top FeRGs in two cell lines are completely different. In the THP-1 cells, Cxcl13 was the gene most significantly induced by both doses of FeNPs. Cxcl13 is a strong humoral immune response gene in various neuroinflammatory diseases [27]. Other FeRGs including Adamdec1, Ebi3, Ifi44l, Clec7a and Ly96 are also related to immune responses. $M m p 9$ plays a critical role in the positive regulation of the apoptotic process [28]. In the THP-1 cells, most of the most strongly repressed FeRGs encode ribosomal proteins (i.e., Rps11, Rplp2, Rpl14, Rpl27a, Rpl37a and Rpl38). The top FeRGs indicate that the FeNPs resulted in strong activation of defense responses and repression of protein synthesis in the THP-1 cells. Importantly, 7 FeRGs were highly induced, and 7 others were repressed by both ldFeNPs and hdFeNPs in the THP-1 cells (Figure 3), indicating that these effects were stable in the THP-1 cells treated with different doses of FeNPs.

In the HepG2 cells, 3 genes, Ifi27, Ifi6I and Tagln, were highly induced by both the ldFeNPs and hdFeNPs (Figure 3). Ifi27 was the gene that was most significantly induced by both ldFeNPs and hdFeNPs. Another Ifi gene, Ifi6, was also significantly induced by both doses of the FeNPs. Ifi27 and Ifi6 are associated with immune responses [29,30]. Ifi27 codes for a mitochondrial protein that contributes to IFN-induced cell death and apoptosis through perturbation of normal mitochondrial function [31]. Tagln, which is associated with cell migration, was also induced genes in the HepG2 cells treated with both doses of FeNPs [32]. Seven genes were among the most significantly repressed genes in the HepG2 cells treated with both ldFeNPs and hdFeNPs. Arnt2 and Etv5 encode DNA-binding transcription factors (TFs). The TF encoded by Arnt 2 acts as a partner for several sensor 

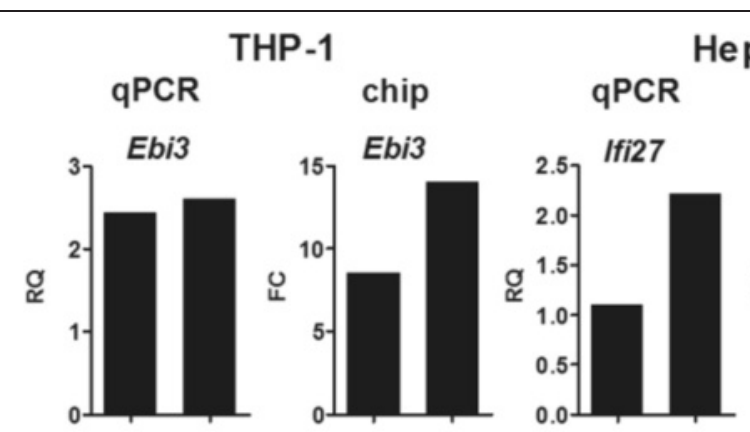

chip
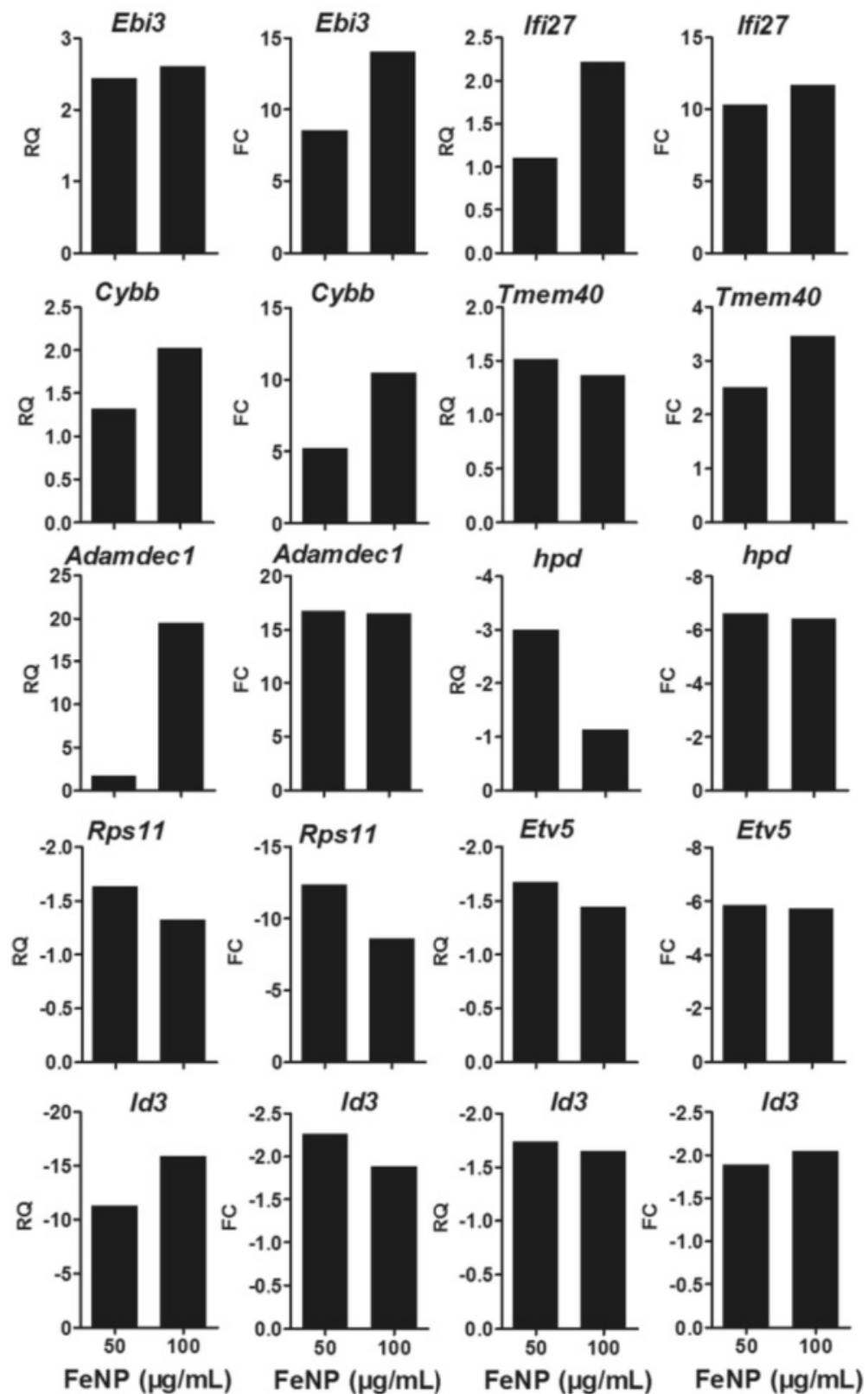

FeNP $(\mu \mathrm{g} / \mathrm{mL})$

FeNP $(\mu \mathrm{g} / \mathrm{mL})$

$\operatorname{FeNP}(\mu \mathrm{g} / \mathrm{mL})$

Figure 2 Quantitative PCR (qPCR) detection of the transcription of genes. The transcription of 5 genes in each type of cells was detected with $\mathrm{qPCR}$. The relative quantification (RQ) of the $\mathrm{qPCR}$ detection was compared with the fold change (FC) of the GeneChip detection.

proteins that bind the regulatory DNA sequences in genes responsive to developmental and environmental stimuli. The TF coded by Etv5 is a member of the ETS family. Hpd encodes an enzyme in the catabolic pathway of tyrosine; KIAA1199 (Cemip) encodes a cell migration-inducing protein; Spink6 encodes a serine protease inhibitor selective for kallikreins; and Frmd3 encodes a putative tumor suppressor protein. Ccnd1 encodes cyclin D1 of the cyclin family, which functions as regulator of the CDK kinases
CDK4 or CDK6, which are required for the cell cycle G1/S transition. Clearly, most of these top repressed genes are involved in cell growth, proliferation and migration.

The genes commonly regulated in both cell lines revealed the common responses of cells to the FeNPs at the gene expression level. A four-way Venn analysis revealed that 2 genes (Ifi27 and $D d x 58$ ) were commonly induced by two doses of FeNPs in these two cell lines (Figure 4A). Eleven genes (Ifi27, Ifi44, Ifit3, Ddx58, 


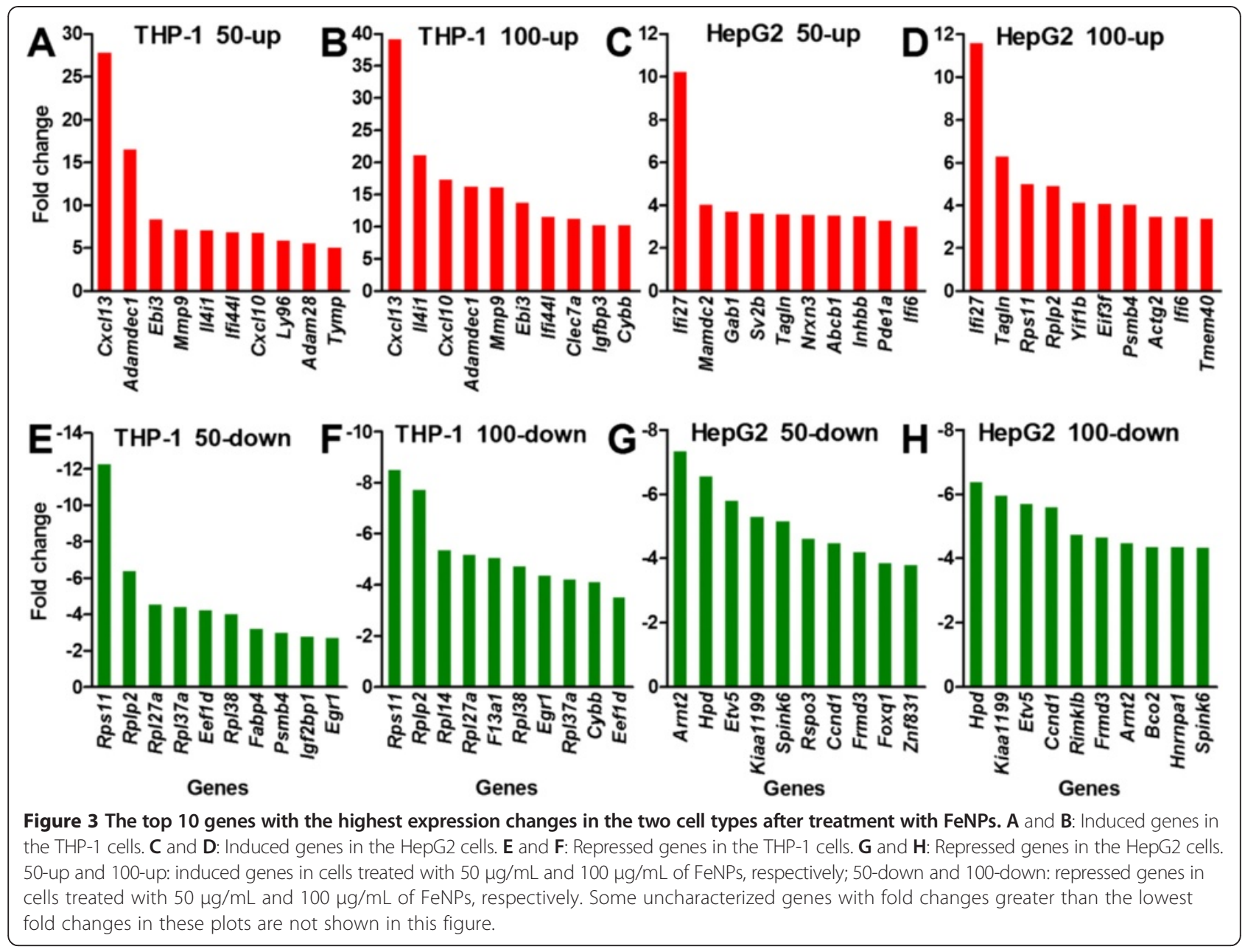

Fbxo16, Parp9, Serpini1, Usp25, Ccne2, Nexn, and Rg9mtd2) were commonly induced by ldFeNPs in both cell lines, and 10 genes (Ifi27, Ifi6, Ddx58, Akap12, Col9a2, Nampt, Narg1, Tmed2, Usp16, and Zcchc2) were commonly induced by hdFeNPs in both cell lines
(Figure 4A). However, no genes were commonly repressed by both doses of FeNPs in both cell lines (Figure 4B). Only one gene (Egrl) was commonly repressed by the hdFeNPs in both cell lines (Figure 4B). Further identification of genes with fold changes greater
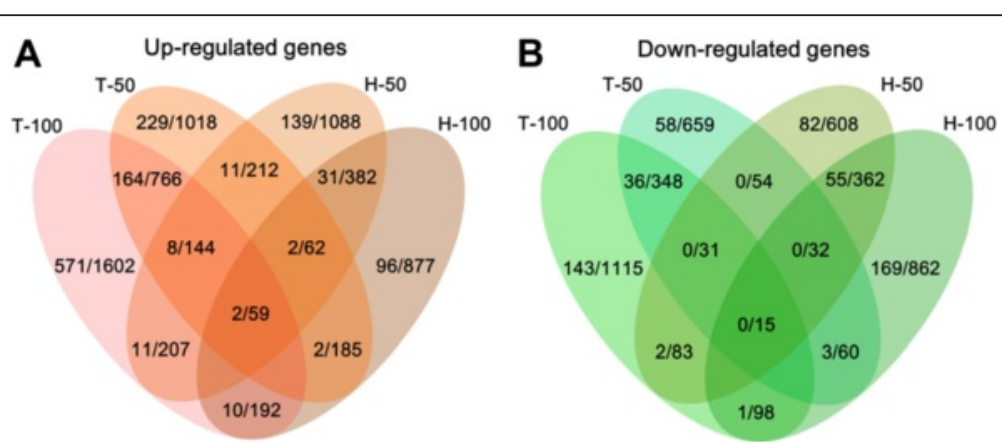

Figure 4 Comparison of FeRGs in the THP-1 and HepG2 cells. A: Comparison of induced genes in the two cell lines. B: Comparison of repressed genes in the two cell lines. Each Venn diagram is divided into four areas labeled as T-50, T-100, H-50 and H-100. T-50 and T-100, THP-1 treated with $50 \mu \mathrm{g} / \mathrm{mL}$ and $100 \mu \mathrm{g} / \mathrm{mL}$ of FeNPs, respectively. H-50 and H-100, HepG2 cells treated with $50 \mu \mathrm{g} / \mathrm{mL}$ and $100 \mu \mathrm{g} / \mathrm{mL}$ of FeNPs, respectively. The number in overlapped area represents the overlapping genes. The numbers before and after the slash represent the genes with fold changes greater than 2 and 1.5 , respectively. 
than 1.5 , but at least in one of the cases, greater than 2.0, revealed that 9 and 4 genes were induced and repressed, respectively by both doses of FeNPs in the two cell lines (Figure 5). Among these genes, Ifi27, Ifi44, Ifi6 and Ifit3 express interferon-induced proteins as a defense response to viruses, and $D d x 58$ is involved in the viral doublestranded (ds) RNA recognition and the regulation of immune response. These genes indicate that the FeNPs induced cellular responses in the treated cells similar to those induced by viruses [33]. Parp 9 and Nexn are associated with cell migration. Ccne 2 belongs to the highly conserved cyclin family and is involved in cell division [34]. Akap12 encodes a cell proliferation-related protein $[35,36]$. The repressed genes, Egr1 and GLI3, encode the $\mathrm{C}_{2} \mathrm{H}_{2}$-type zinc-finger proteins of the EGR family, which play roles in cell proliferation [37,38]. Id3 is associated with cell growth $[39,40]$.

\section{Cluster analysis of FeNP-responsive genes}

The four-way Venn analysis found that only 2 genes were commonly regulated by both doses of FeNPs in the two cell lines (Figure 4). To identify additional commonly regulated genes in these two cell lines, the genes differentially expressed under at least one treatment in each of two cell types were identified. As a result, 55 commonly regulated genes were found (Figure 6). The hierarchical clustering analysis revealed that these genes were classified into four clusters. Clearly, some genes were consistently induced or repressed in both types of cells (Clusters A and D), whereas some genes were inversely regulated in the two cell lines (Clusters B and C). The former reveals the cell-independent effects, whereas the latter reveals the cell-specific effects of the FeNPs on the gene expression in these two cell lines.

\section{Functional annotation of FeNP-responsive genes}

The GO analysis revealed that the inflammatory, defense and immune responses, and the responses to stress, wounding, external stimuli, biotic stimuli, viruses, and other organisms were most significantly enriched in the THP-1 cells (Figure 7). The inflammatory and defense responses were the two biological processes most significantly enriched among the genes induced by both ldFeNPs and hdFeNPs in the THP-1 cells (Figure 7). In combination with the common enrichment of the responses to viruses in the cells treated with both doses of FeNPs and the similar particulate structure of FeNPs and virus, it seems that the THP-1 cells recognized FeNPs as viruses and responded with virus-like cellular effects. Similar responses were also found in the RAW264.7 cells [10], revealing that the virus-like cellular effects are a common cytotoxic response to the FeNPs in the monocyte-macrophage system. In addition, the hdFeNPs activated many more genes of these response-related biological processes in the THP-1 cells (Figure 7), indicating that the hdFeNPs induced more intense virus-like cellular effects. The exacerbated cytotoxicity induced by the hdFeNPs is also indicated by the activation of the biological process of cell death in cells treated with the hdFeNPs (Figure 7), which agrees with the significant apoptosis of the THP-1 cells that resulted from the treatment with $100 \mu \mathrm{g} / \mathrm{mL}$ of the same nanoparticles [7]. In the HepG2 cells, several response-related biological processes were also enriched among the genes induced by ldFeNPs, similar to those observed in the Hepa1-6 cells treated with the hdFeNPs [10].

To evaluate the distribution of FeRGs among various biological processes, the GO terms were classified into five categories at the first level, and the FeRGs belonging to each category were counted. The results demonstrate that the hdFeNPs significantly increased the induced genes in each category in the THP-1 cells but decreased the induced genes in each category in the HepG2 cells (Figure 8). This is completely different from the effects observed in the mouse cell lines. Specifically the hdFeNPs decreased the numbers of the induced genes in these categories in the RAW264.7 cells but increased the numbers of the induced genes in these categories in the Hepa1-6 cells [10]. However, the hdFeNPs increased the numbers of the repressed genes in each category in both two human (Figure 8) and mouse cell lines [10]. These data reveal that two human cell lines responded to the two doses of FeNPs with the different patterns of gene expression. These data also demonstrate that the FeNPs induced
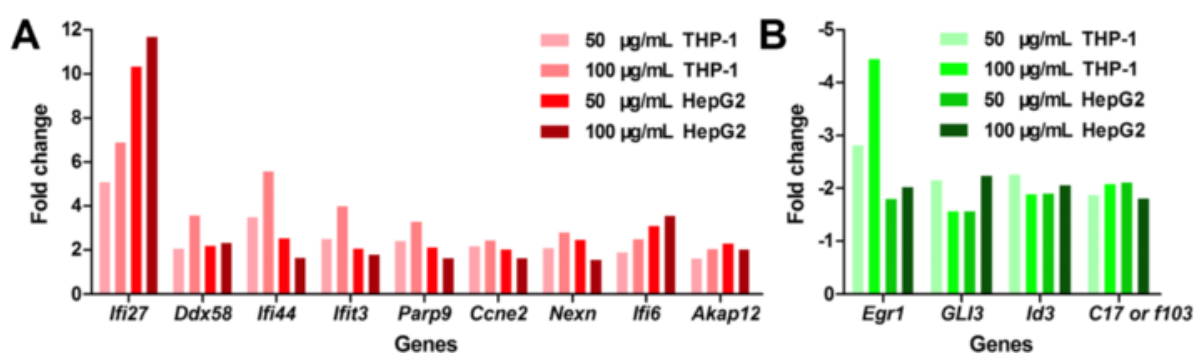

Figure $\mathbf{5}$ Commonly regulated genes in the THP-1 and HepG2 cells. A: The induced genes and their expression levels in the two types of cells treated with two doses of FeNP. B: The repressed genes and their expression levels in the two types of cells treated with two doses of FeNP. 


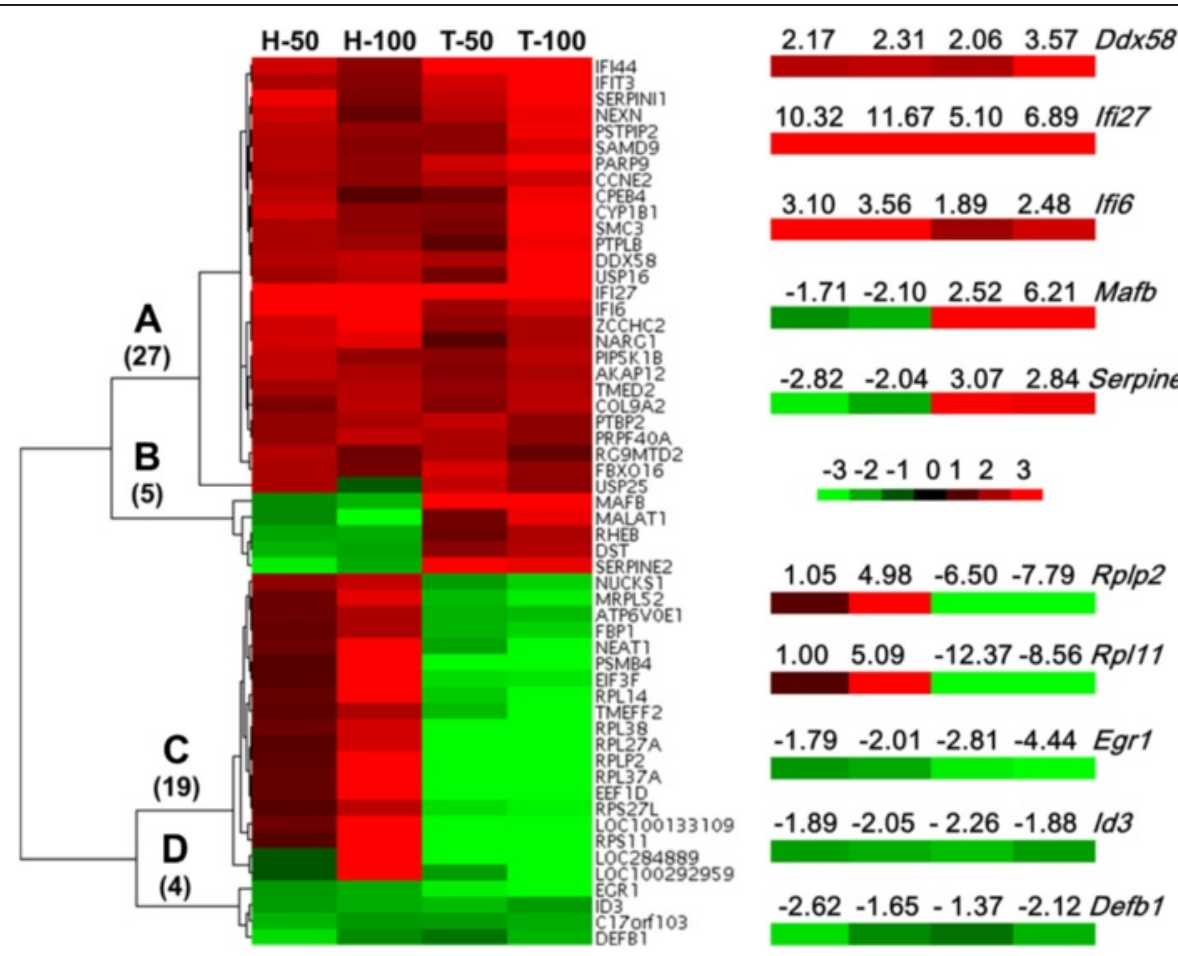

Figure 6 Cluster analysis of genes. Fifty-five FeRGs from the two cell lines were clustered according to their expression levels using a hierarchical clustering. The heatmap was drawn with Java TreeView. Red and green represent up- and down-regulation, respectively. The color depth reflects the expression level between -3 and +3 (marker). The numbers of genes in Clusters $\mathbf{A}$ to $\mathbf{D}$ are shown in parentheses. The fold changes of 10 representative genes in four clusters are shown in the zoomed images. T-50, H-50, T-100, and H-100, see Figure 4.

different forms of nanotoxicity in cell types with different biological functions.

To identify the biological processes associated with the genes that were consistently regulated by two doses of FeNPs in two cell lines (clustered in Figure 6), a new GO analysis was performed with these genes. The results revealed that 4 biological processes were enriched mainly by two commonly repressed genes (EgrI and Id3), whereas 16 biological processes were enriched by 13 genes that were inversely regulated in the two cell lines (Figure 9). The 16 biological processes were all clearly associated with protein translation and were primarily determined by 7 nucleosomal proteins (Rpl14, Rpl27a, Rplp2, Rpl37a, Rps11, Rpl38, and Rps27l), a translation initiation factor (Eif3f) and a translation elongation factor (Eef1d). These genes were induced in the HepG2 cells but repressed in the THP-1 cells, indicating that the FeNPs significantly induced protein production in the HepG2 cells but repressed this process in the THP-1 cells. These results are consistent with the results of our recent evaluation of cell viability, which indicated that the ldFeNPs significantly $(\mathrm{p}<0.05)$ and hdFeNPs very significantly $(\mathrm{p}<0.01)$ decreased the viability of the THP-1 cells, but neither the ldFeNPs nor the hdFeNPs affected the viability of the HepG2 cells (not shown). Interestingly, a gene encoding a mitochondrial ribosomal protein L52 (Mrpl52) was also significantly repressed in the THP-1 cells but induced in the HepG2 cells by the FeNPs (Figure 9), indicating that both cytoplasmic and mitochondrial protein production were significantly affected by the FeNPs. In addition, one biological process (nervous system development) was represented by 3 genes repressed in HepG2 but induced in THP-1 (Serpine2, Mafb and Dst). Such inverse regulation of a biological process demonstrates the typical cellspecific effects of FeNPs.

\section{Pathway analysis of FeNP-responsive genes}

The most significantly $(\mathrm{p}<0.05)$ enriched KEGG pathways are shown in Figure 10. In the THP-1 cells, the Toll-like receptor (TLR) signaling pathway is significantly enriched by the FeRGs that were induced by both ldFeNPs and hdFeNPs. TLRs are membrane-bound receptors expressed on innate immune cells such as macrophages and dendritic cells, which generate innate immune responses [41,42]. The TLR signaling pathway was reported to be activated by the FeNPs in RAW264.7 cells [9], and by ceramic $\left(\mathrm{TiO}_{2}, \mathrm{SiO}_{2}\right.$ and $\left.\mathrm{ZrO}_{2}\right)$ and metallic (cobalt) nanoparticles in a human myelomonocytic cell line (U-937) [41]. In addition, the RIG-I-like receptor and chemokine signaling pathways were significantly enriched by the FeRGs induced by both ldFeNPs and hdFeNPs. The cytosolic DNA-sensing pathway and the 


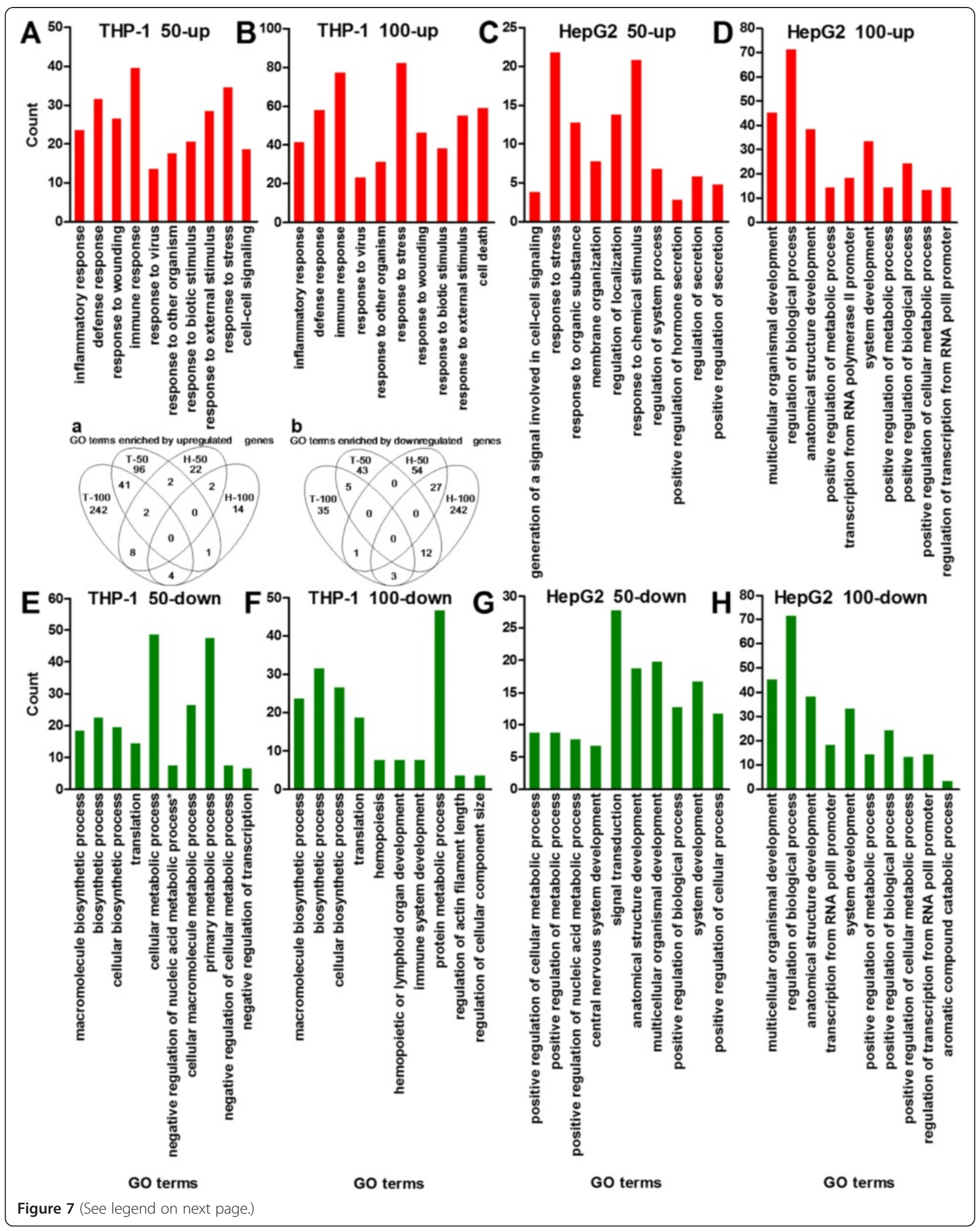


(See figure on previous page.)

Figure 7 Top $10 \mathrm{GO}$ terms enriched by induced and repressed genes in the two cell lines. A and B: GO terms enriched by the induced genes in the THP-1 cells. C and D: GO terms enriched by the induced genes in the HepG2 cells. $\mathbf{E}$ and $\mathbf{F}$ : GO terms enriched by the repressed genes in the THP-1 cells. $\mathbf{G}$ and $\mathbf{H}$ : GO terms enriched by the repressed genes in the HepG2 cells. *Negative regulation of nucleobase, nucleoside, nucleotide and nucleic acid metabolic process. Polll, polymerase II. The p values for all GO terms are less than 0.05. In each plot, the GO terms were aligned from left to right according to their $p$ values from low to high. 50-up, 100-up, 50-down, and 100-down, see Figure 3. Inset: Venn analysis of all GO terms enriched by the induced genes (a) and repressed genes (b) in the THP-1 and HepG2 cell lines. T-50, H-50, T-100, and $\mathrm{H}-100$, see Figure 4. The numbers in overlapped areas represent the overlapping GO terms.

NOD-like receptor signaling pathway were significantly enriched by the FeRGs induced by hdFeNPs. All these pathways are known to play critical roles in immunological responses [43,44]. Additionally, more genes in these pathways were induced by hdFeNPs. These data indicate that the hdFeNPs induced more intense immunological responses than the ldFeNPs in the THP-1 cells. This is consistent with the results of the GO analysis that revealed that the biological process of immune response was consistently highly enriched by the FeRGs induced by both ldFeNPs and hdFeNPs; however, the ldFeNPs induced 40 immune response-related FeRGs, but the hdFeNPs induced 78 immune response-related FeRGs (Figure 7). The more intense reactions of the cells

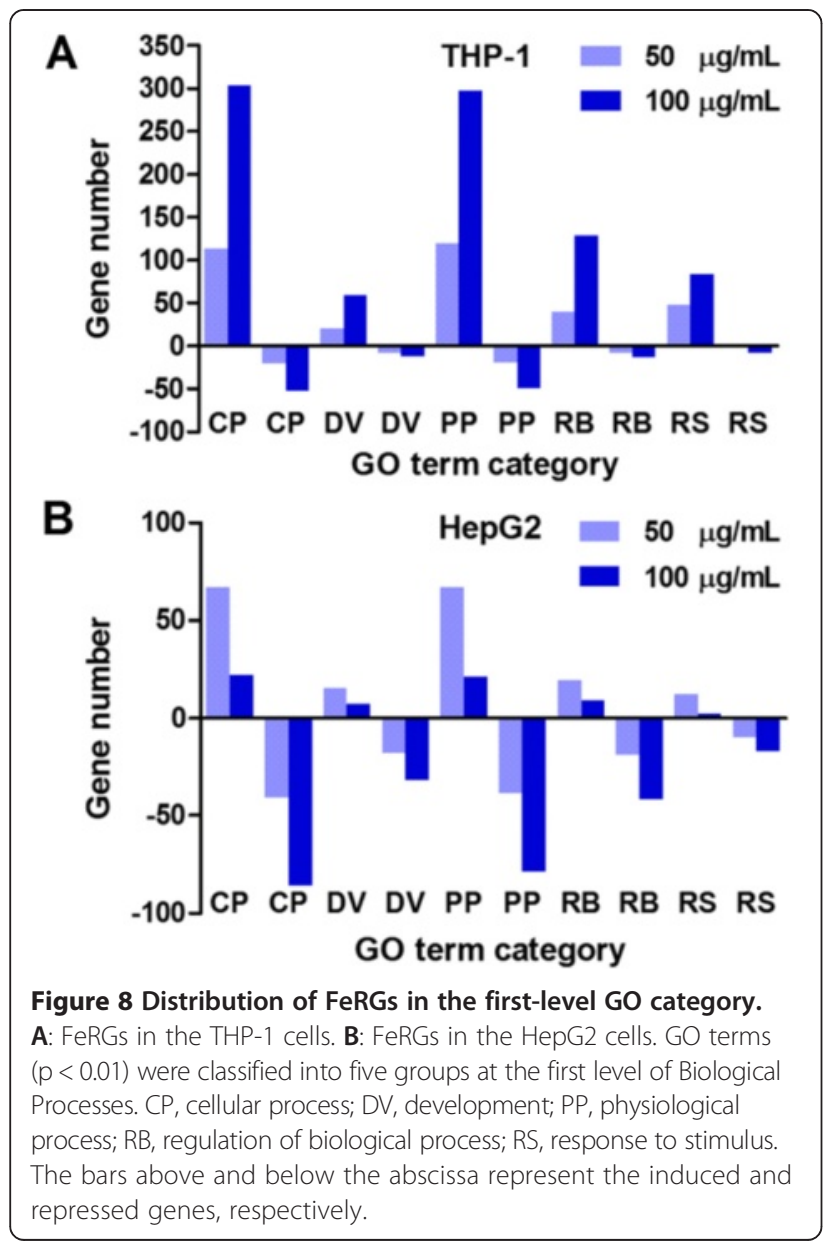

to the hdFeNPs were also demonstrated by the significant activation of five human disease pathways by hdFeNPs, including the Leishmaniasis, Rheumatoid arthritis, Staphylococcus aureus infection, Toxoplasmosis, and Malaria pathways (Figure 10).

It is interesting that the hepatitis $C$ pathway is significantly enriched by the FeRGs induced by both the ldFeNPs and hdFeNPs in the THP-1 cells (Figure 10). Moreover, more genes in this pathway were induced by the hdFeNPs (Figures 10 and 11). In this pathway, the gene Oas1, Oas2 and Oas3 were induced by both doses of FeNPs (Figure 11). These genes encode the 2',5' -oligoadenylate $(2-5 \mathrm{~A})$ synthetases, enzymes that play essential roles in the innate immune response to viral infection $[45,46]$. In this pathway, the gene $D d x 58$ (coding RIG-I) was also induced by both ldFeNPs and hdFeNPs (Figure 11). RIG-I functions as a pattern recognition receptor that is a sensor for viruses such as hepatitis $\mathrm{C}$ virus. The activation of RIG-I-like receptor signaling pathway can induce the production of interferon [47], which is supported by the significant overexpression of many interferon-related genes, including Ifi27, Ifi6, Ifi16, Ifi35, Ifi44, Ifi44l, Ifit1, Ifit2, Ifit3, Ifit5, Ifitm1, Ifih1, Isg20, and Irf7. The GO analysis also revealed that the biological process of response to viruses was significantly enriched by the FeRGs induced by the two doses of FeNPs in the THP-1 cells (Figure 7). These data indicate that the FeNPs act on the THP-1 cells similarly to the responses to viruses including the hepatitis $\mathrm{C}$ virus. This is also in consistent with the fact the hydrodynamic size of the FeNPs (41 nm) (Figure 1) used in this study is similar to the size of the hepatitis $B$ virus particles $(42 \mathrm{~nm})$.

In the THP-1 cells, only the ribosomal pathway was significantly enriched by the FeRGs repressed by both ldFeNPs and hdFeNPs (Figure 10E and F). In this pathway, 10 genes coding for ribosome proteins, including Rps27l, Rplp2, Rps11, Rps19, Rpl14, Rpl27, Rps10, Rpl38, $R p l 37 a$, and $R p l 27 a$, were commonly repressed by both doses of FeNPs. The extensive repression of these genes suggests that the FeNPs significantly inhibited protein production in this cell. The similar repression of ribosomal pathway was also found in the zebra fish embryos exposed to silver nanoparticles [22]. However, the ribosomal pathway was also the most significantly enriched 


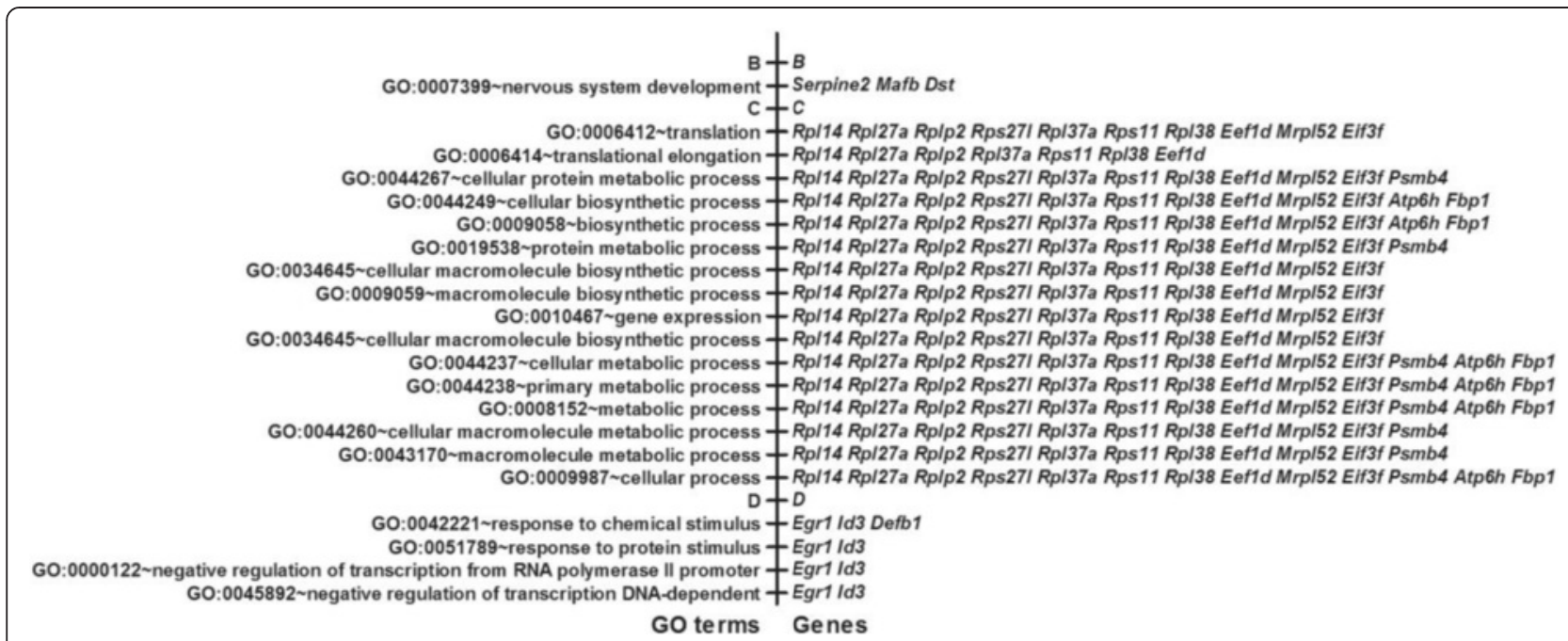

Figure 9 GO analysis of the FeRGs with different expression patterns. B, C and D, genes in Clusters B, C and D (Figure 6), and their associated $\mathrm{GO}$ terms. The $p$ values for all $\mathrm{GO}$ terms are less than 0.05. An alias of Atp6h is Atp6r0e1.

by the FeRGs induced by the hdFeNPs in the HepG2 cells (Figure 10D). In this cell type, 11 genes coding ribosome proteins, including Rps27l, Rplp2, Rps11, Rps19, Rpl14, Rpl27, Rps10, Rpl38, Rpl37a, Rpl27a, and Rps6, were significantly induced by hdFeNPs. Except for Rps6, these genes are identical to those altered in the THP-1 cells. These data indicate that the ribosomal pathway was inversely regulated by the FeNPs in two cell lines due to the opposite regulation of a same set of genes coding ribosome proteins. Such differential regulation of a set of genes and their involved pathway demonstrate a typical form of cell-specific toxicology of a nanomaterial.

In the HepG2 cells, no pathway was significantly enriched by the genes induced by the ldFeNPs (Figure 10C). However, 8 pathways were significantly enriched by the genes induced by the hdFeNPs (Figure 10D). These pathways are mainly associated with cellular metabolism. The ribosome pathway was highly enriched by the FeRGs induced by the hdFeNPs. Proteasomes are responsible for protein metabolism (proteolysis). Three pathways are responsible for the metabolism of other nitrogen-containing materials, including the nitrogen, nicotinate and nicotinamide, and alanine, aspartate and glutamate pathways. The insulin signaling pathway is responsible for glucose metabolism. In addition to these metabolism-related pathways, the ECM-receptor interaction and calcium signaling pathways were also significantly enriched by the genes induced by hdFeNPs in HepG2. Two extracellular matrix (ECM) macromolecules, vitronectin (encoded by $V t n$ ) and collagen (encoded by Col6a1), which benefit cell proliferation and migration, were significantly induced by the hdFeNPs. Four genes (P2rx4, Tnnc1, Pde1a, and Erbb4) in the calcium signaling pathway, which is closely associated with cell adhesion, were significantly induced by hdFeNPs. Therefore, the pathways enriched by the induced genes in the HepG2 cells were mainly associated with cellular metabolism, proliferation, migration and adhesion.

In the HepG2 cells, two pathways, TGF-beta signaling and focal adhesion, were significantly enriched by the FeRGs repressed by both the ldFeNPs and hdFeNPs (Figure 10G and H). Furthermore, more genes in these two pathways are repressed by the hdFeNPs, indicating that the hdFeNPs exert greater effect on the HepG2 cells than the ldFeNPs. This dose-dependent effect can be observed in the THP-1 cells (Figure 10A and B). Importantly, the TGF-beta signaling pathway was most significantly enriched by the genes repressed by both doses of the FeNPs in the HepG2 cells. This pathway is the most important pathway responsible for cell viability. Furthermore, a wide spectrum of cellular functions such as proliferation, apoptosis, differentiation and migration are regulated by TGF- $\beta$ family members [48]. In the HepG2 cells, 5 (including $I d 1$, Id2, Bmp6, Smad9, and Tgfb1) and 7 (including Id1, Id2, Id3, Bmp6, Smad6, Smad7, and $S m a d 9)$ genes in this pathway were repressed by the ldFeNPs and hdFeNPs, respectively. Smad6, Smad7 and Smad9 encode proteins of the SMAD family that act as signal transducers and transcriptional modulators [49]. Id 1 , Id 2 and Id 3 encode helix-loop-helix (HLH) proteins that function as dominant-negative regulators of basic HLH (bHLH) transcription factors by forming inactive heterodimers with intact bHLH. ID proteins play important roles in control of cell growth, differentiation and tumorigenesis [50,51]. The down-regulation of these genes can promote cell growth and increase the risk of tumorigenesis [52]. These data suggest that the FeNPs 


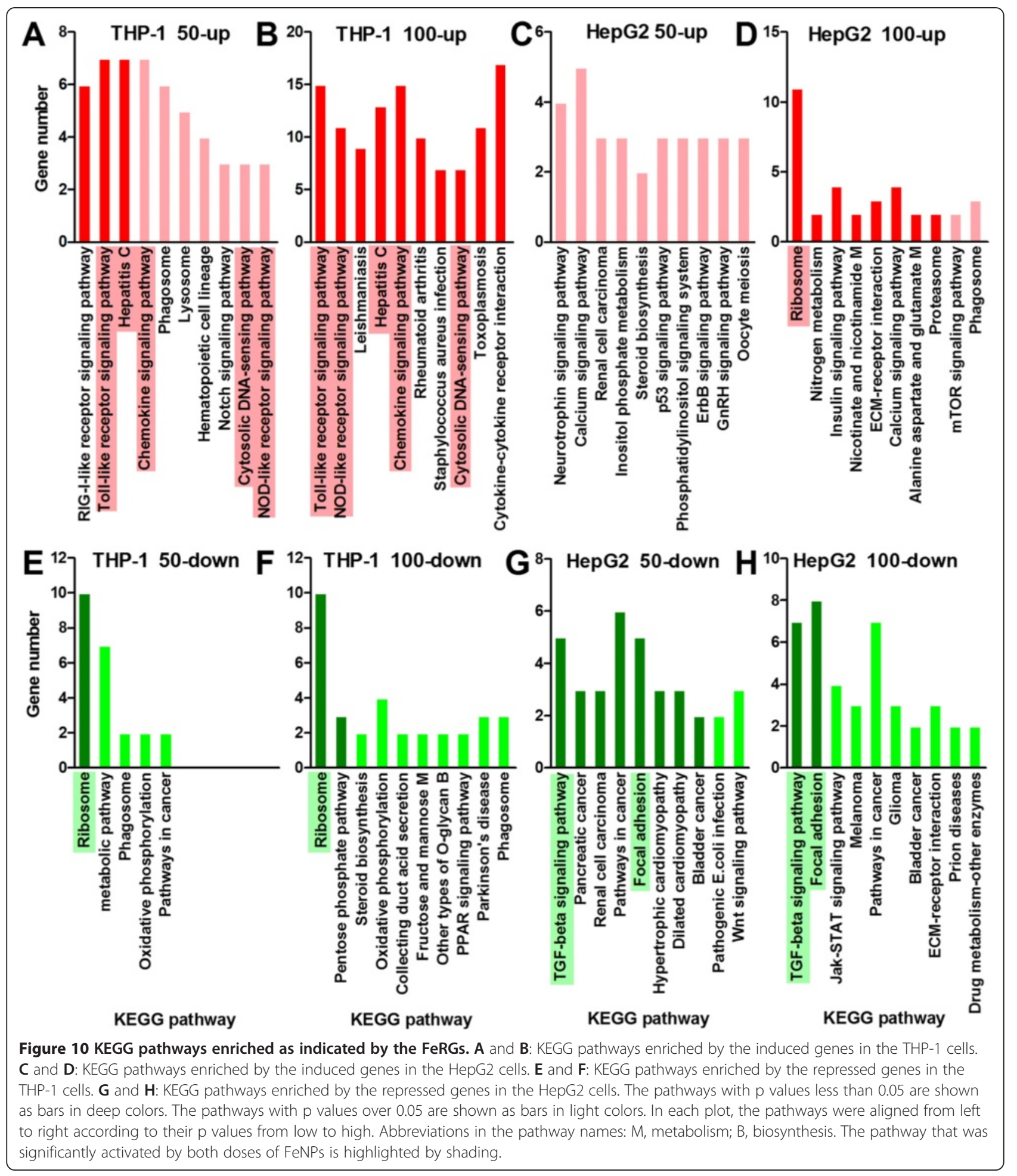

may stimulate the growth of the HepG2 cells. This is supported by the results of cell viability assay, which revealed that neither the ldFeNPs nor the hdFeNPs affected the viability of the HepG2 cells, and the hdFeNPs even enhanced cell viability (106.4\% of the untreated control cells) (not shown). This is also in agreement with the activation of the ribosomal pathway and several metabolism-related pathways by the hdFeNPs in this cell.

The focal adhesion pathway was also significantly enriched by the FeRGs repressed by both the ldFeNPs and hdFeNPs in the HepG2 cells. The pathway plays an 
HEPATITIS C

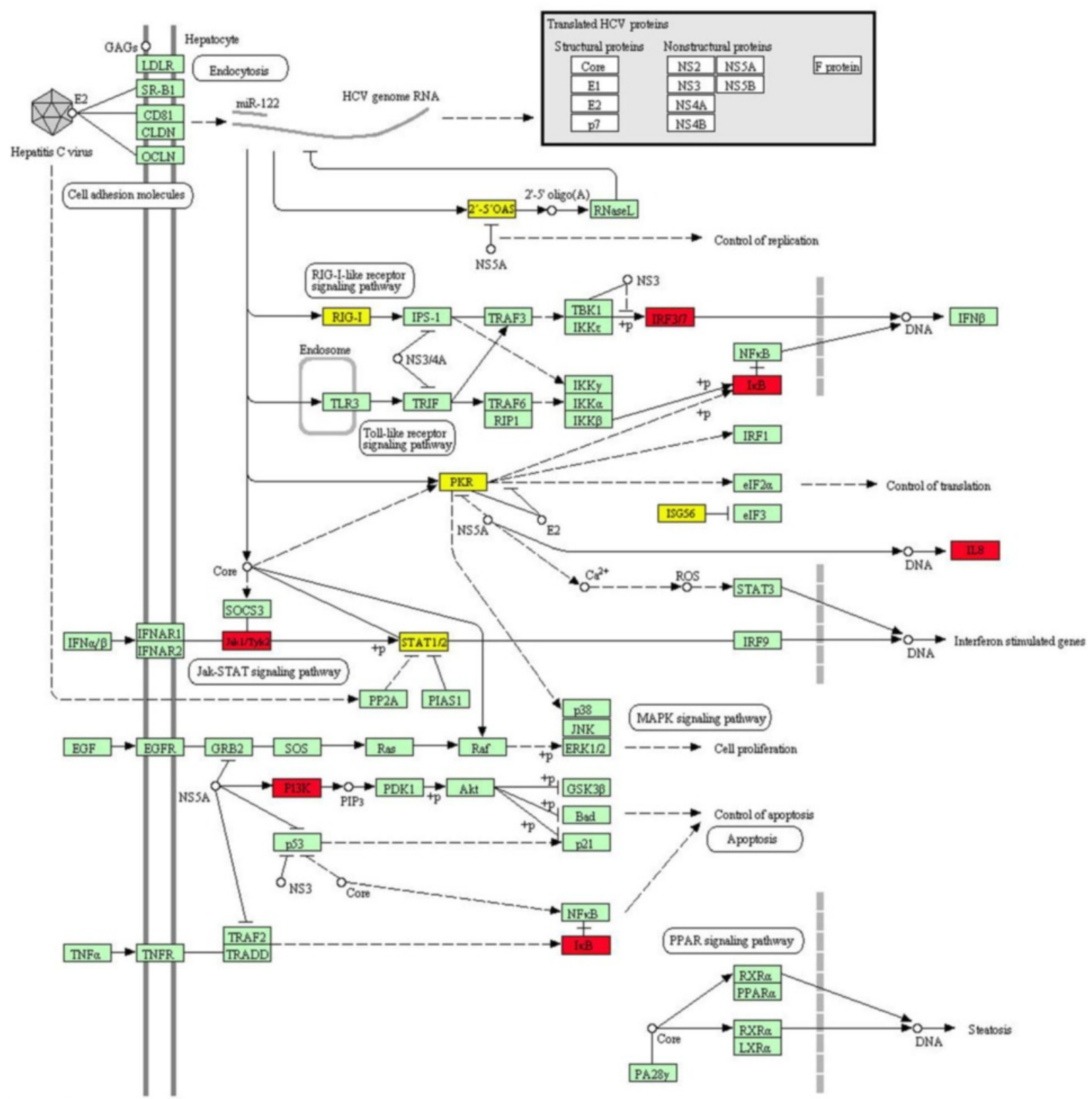

$051601115 / 13$

Figure 11 KEGG pathway of hepatitis C in the FeNP-treated THP-1 cells. The genes in red refer to the FeRGs induced by $100 \mu \mathrm{g} / \mathrm{mL}$ of FeNPs. The genes in yellow refer to the FeRGs induced by both $50 \mu \mathrm{g} / \mathrm{mL}$ and $100 \mu \mathrm{g} / \mathrm{mL}$ of FeNPs. Abbreviations for the KEGG parameters can be found on the KEGG pathway webpage.

essential role in important biological processes including cell motility, cell proliferation, cell differentiation, regulation of gene expression, and cell survival [53]. In the HepG2 cells, 4 (Ccnd1, Fyn, Fn1, Itgb5, and Vegfc) and 8 (Cond1, Fyn, Fn1, Itgb5, Col3a1, Vav3, Igf1r, and Egfr) genes of this pathway were significantly repressed by the ldFeNPs and hdFeNPs, respectively. The down-regulation of these important cell adhesion-related genes suggests that the growth and mobility of the cells were enhanced by the FeNPs, which is consistent with the slight increase of cell viability and activation of ribosomal pathway. The enhancement of cell growth and mobility may also increase the risk of tumorigenesis. This is supported by the enrichment of the TGF-beta signaling pathway and several 
cancer-related pathways including those associated with pancreatic cancer, renal cell carcinoma, and bladder cancer in the HepG2 cells (Figure 10G). In the HepG2 cells, these cancer-related pathways are mainly enriched by the genes Ccnd1, Vegfc, Tgfb1, Arnt2, Fn1, and Fzd10.

In the pathway analysis, it was found that the phagosome pathway was also enriched by several genes in both the THP-1 and HepG2 cells. In the THP-1 cells, this pathway was enriched by 6 genes induced by ldFeNPs (Atp6v1h, Hla-Dra, Clec7a, Cd14, Cd36, and Cybb), 2 genes repressed by ldFeNPs (Calr and Atp6v0e1), and 3 genes repressed by hdFeNPs (Calr, Atp6v0e1, Atp6v1c2) (Figure 9D and E). In the HepG2 cells, this pathway was enriched by 3 genes induced by hdFeNPs (Calr, Atp6v0e1, and Ctss) (Figure 10F). Clearly, the genes Calr and Atp6v0e1 were commonly regulated by the FeNPs in both cell lines; however, the expression of these genes was inversely regulated in the two cell lines. This indicates once again the inverse toxicity of the FeNPs in cells of different functions. However, these genes demonstrated the endocytotic activity of two cells to the FeNPs, which agrees with the previous reports that the FeNPs were internalized into cells by endocytosis [54-57]. In addition to the phagosome pathway, the lysosome and notch signaling pathways were also enriched by the genes induced by ldFeNPs in the THP-1 cells (Figure 10A). In this cell line, the lysosomal pathway was enriched by 5 genes (Atp6v1h, Laptm4b, Ap1s3, Ctsh, and Lamp3), and the notch signaling pathway was enriched by 3 genes (Maml2, Lfng, and Dtxy) (Figure 10A). These results agree with many previous reports that FeNPs are accumulated into the lysosomes $[13,42]$.

\section{Comparison of human cells with mouse cells}

The effects of the same FeNPs at the same two doses on the gene expression profiles of two mouse cell lines (RAW264.7 and Hepa1-6) were recently investigated by our lab [10]. To identify the effects of the FeNPs on the cells of different species, this study used the human equivalents of mouse cells. THP-1 and RAW264.7 are cell lines of monocyte-macrophage system, whereas HepG2 and Hepa1-6 are hepatoma cell lines. The genes with changes $\geq 1.5$-fold, the GO terms, and the KEGG pathways for the four cell types were systematically compared. The genes with one or two changes $\geq 2$.0-fold among the compared cells were identified as the common genes. The common genes, GO terms, and KEGG pathways identified in the two monocyte-macrophage cell lines, the two hepatoma cell lines, and all four cell lines were shown in Figure 12.

Although some pathways were significantly $(\mathrm{p}<0.05)$ enriched in a manner common to both human and mouse cells, the FeRGs involved in these pathways were quite different. For example, in 3 commonly enriched pathways of the two liver cells (Figure 12B), only the focal adhesion pathway shared a gene (Col3a1) between the human and mouse cells. In the 8 pathways enriched in common to the two types of blood cells (Figure 12A), only two genes ( $\mathrm{Ccl} 4$ and $\mathrm{Ccl} 5$ ) were shared by the human and mouse cell lines. The Ccl4 and $\mathrm{Ccl} 5$ genes shared by two cell lines are involved in the TLR signaling pathway, chemokine signaling pathway, and cytosolic DNA-sensing pathway. The gene Ccl4, shared by two cells, is in the cytokine-cytokine receptor interaction pathway. The gene Ccl5 shared by two cells is in the rheumatoid arthritis pathway. Additionally, it was found that the most common pathways were enriched by the induced or repressed genes in the cells of both species. For example, the TLR signaling pathway is enriched by the induced genes in both human and mouse cells, but the focal adhesion is enriched by the repressed genes in both human and mouse cells (Figure 12A and B). However, two other pathways were inversely regulated in cells of two species. Specifically, the alanine, aspartate and glutamate metabolism and Leishmaniasis pathways were enriched by repressed genes in mouse cells but the induced genes in human cells (Figure 12A and B).

The GO analyses of the common genes revealed that four biological processes were significantly $(\mathrm{p}<0.05)$ enriched in the two blood cell types. These biological processes are mainly related to the responses of the cells to stimuli and stress (Figure 12D). The GO analyses of the common genes revealed that no biological processes were significantly $(\mathrm{p}<0.05)$ enriched in the two liver cells. However, the biological processes of cell adhesion and cell-matrix adhesion were enriched by three genes, Ctgf, Tgfbi and Vtn, in the two liver cells. It seems that the THP-1 cells are more sensitive to the FeNPs than the RAW264.7 cells because in the 8 common pathways (Figure 12A), many more genes were induced by the FeNPs in the THP-1 cells (Figure 12E). For instance, in the TLR signaling pathway, only two genes $(\mathrm{Ccl} 4$ and Ccl5) were significantly induced by the FeNPs in the RAW264.7 cells, however, as many as 15 genes $(\mathrm{Ccl} 4$, Ccl5, Cxcl10, Cxcl11, Cd14, Cd40, Cd86, Il8, Il1b, Nfkbia, Stat1, Irf7, Ly96, Tlr8, and Pik3r1) were significantly induced by the FeNPs in the THP-1 cells (Figure 12E).

Identification of the common genes revealed that only the $I d 3$ gene was commonly regulated by the FeNP in all four cell lines (Figure 12F). Id3 (inhibitor of DNA binding 3) was the second most significantly repressed gene in the RAW264.7 cells [10]. Previous studies have demonstrated that ID3 is a redox-sensitive signaling molecule $[39,40,58]$. Id 3 and Gklf were identified as two differentially regulated redox-sensitive genes in vascular smooth muscle cells (VSMC) [40]. Id3 was induced by xanthine/ xanthine oxidase $(\mathrm{X} / \mathrm{XO})$ but repressed by $\mathrm{Fe}^{3+} \mathrm{NTA}$ (H-Fe). Conversely, Gklf was repressed by $\mathrm{X} / \mathrm{XO}$ but 


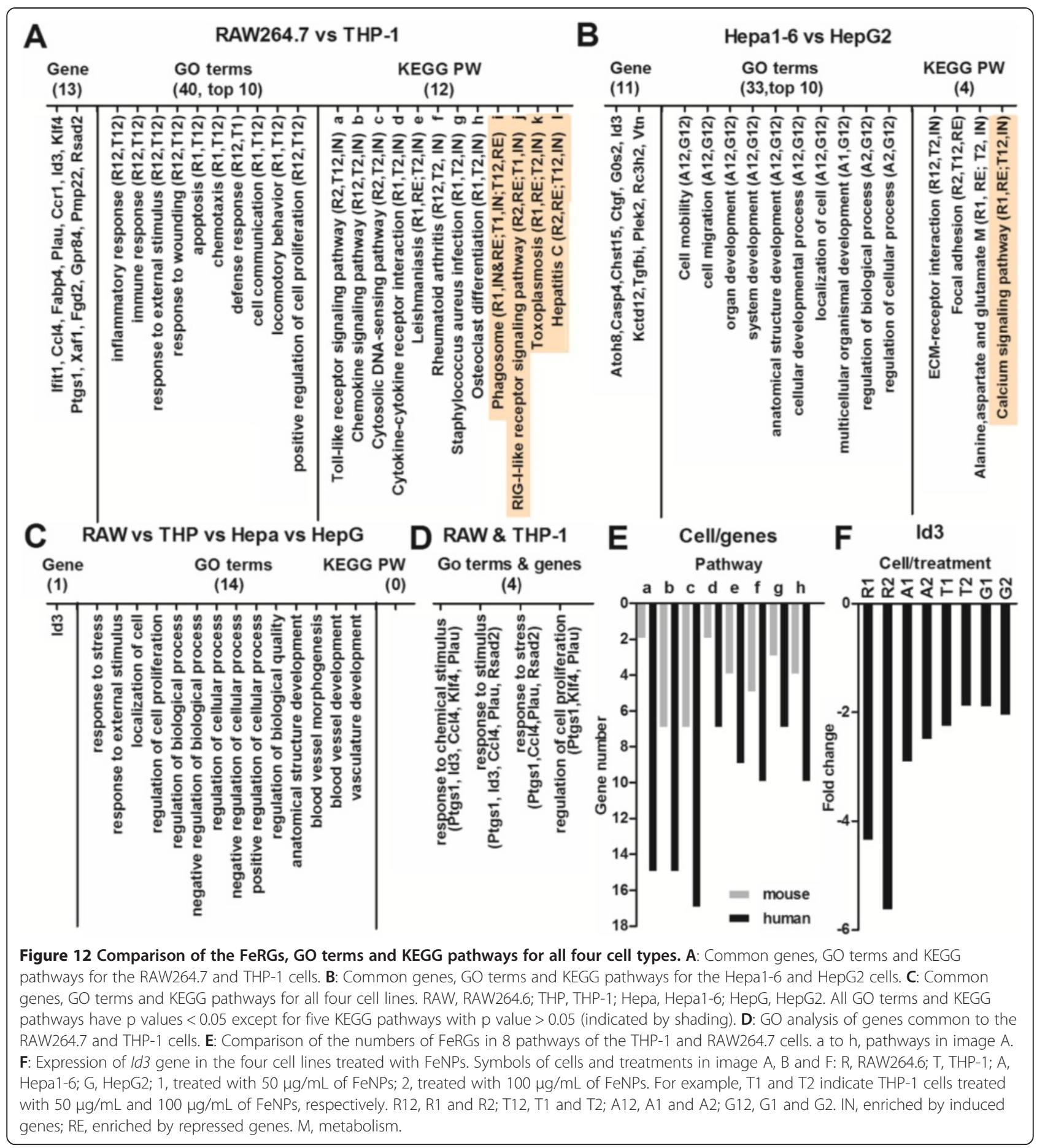

induced by $\mathrm{H}-\mathrm{Fe}$ [40]. It was found that Gklf could reduce the $I d 3$ expression by binding to its promoter [40]. It is well known that FeNPs have redox activity and could result in oxidative stress in cells [59-63]. Therefore, the redox-sensitive down-regulation of $I d 3$ in VSMC is perfectly in agreement with the significant down-regulation in common among the four cell lines treated with two doses of FeNPs. These data suggest that the FeNPs used in this study induced the common down-regulation of $I d 3$ gene in the four cell lines by its redox activity in cells. These data also show that the down-regulation of the $I d 3$ gene is a general and sensitive biomarker of the nanotoxicity of the FeNPs, which demonstrates that the redox activity of FeNPs and the resulted oxidative stress are the most significant and prevalent form of the cellular toxicity of the FeNPs. In 
addition, because Id 3 codes for the ID3 protein of the HLH transcription factor family, which inhibits transcription by forming nonfunctional dimers with other bHLH transcription factors, its significant down-regulation by FeNP suggests that FeNP may disturb the normal biological processes, including cell growth [39], cell differentiation [51], cell apoptosis [64,65], and tumorigenesis [66-68] that are controlled by this transcription factor.

\section{Conclusion}

This study investigated the effects of an 11-nm DMSAcoated magnetite FeNP on the gene expression profiles of two human cell lines, THP-1 and HepG2. It was found that the expression of hundreds of genes was significantly changed by a 24-h treatment of the FeNPs at two doses, $50 \mu \mathrm{g} / \mathrm{mL}$ and $100 \mu \mathrm{g} / \mathrm{mL}$ in the two types of cells. By identifying the FeRGs and annotating their functions, this study characterized the general and cellspecific effects of the FeNPs on two cells at the gene, biological process and KEGG pathway levels. At the doses used, the overall effects of the FeNPs in the THP1 cells was the induction of various responses and repression of protein translation, but in the HepG2 cells these particles promoted cell metabolism, growth and mobility. This study also characterized the common genes, biological processes and pathways effected by the FeNPs in two human and two mouse cell lines, and identified $I d 3$ as a nanotoxicity biomarker of FeNPs.

\section{Methods}

\section{Reagents and cells}

The DMSA-coated magnetite FeNPs were supplied by the Gu's lab of Southeast University (Nanjing, China) [69]. HEPES and glutaraldehyde were purchased from Sigma Aldrich (St. Louis, MO, USA). The Trizol reagent and the DMEM cell culture medium were purchased from Invitrogen Gibco (Carlsbad, CA, USA). The Human Genome U133 Plus 2.0 GeneChips ${ }^{\oplus}$ microarrays were purchased from Affymetrix (Santa Clara, CA, USA). The Reverse Transcriptase Kit was purchased from TaKaRa (Dalian, China). The Fast SYBR Master Mix was purchased from Applied Biosystem (Grand Island, NY, USA). The THP-1 and HepG2 cells were purchased from the China Center for Type Culture Collection (Shanghai, China).

\section{Characterization of nanoparticles}

The DMSA-coated magnetite FeNPs were synthesized by thermal decomposition [69]. The size and dispersibility of the FeNPs were evaluated using a transmission electron microscope (JEM-2100). The hydrodynamic size distribution of the FeNPs was analyzed with a submicron particle analyzer (Beckman Coulter N4 Plus). The surface charge of the FeNPs was measured with a zeta potential analyzer (Beckman Coulter Delsa 440SX).

\section{Exposure of cells to nanoparticles}

The suspension of the FeNPs in water was sterilized by filtration through a $0.22-\mu \mathrm{m}$ membrane. The human cells were cultured in the DMEM cell culture medium supplemented with $10 \%$ fetal calf serum, 100 units $/ \mathrm{mL}$ penicillin, $100 \mu \mathrm{g} / \mathrm{mL}$ streptomycin and $10 \mathrm{mM}$ HEPES in a humidified $5 \% \mathrm{CO}_{2}$ atmosphere at $37^{\circ} \mathrm{C}$. To treat the cells with FeNPs, the cells were seeded in plates or flasks and cultivated overnight, and the culture medium was then replaced with fresh medium containing one of two concentrations (50 and $100 \mu \mathrm{g} / \mathrm{mL}$ ) of FeNPs. The cells were cultivated for $24 \mathrm{~h}$ longer. To detect the cellular internalization of FeNPs, the cells were stained with Prussian blue as previously described [7].

\section{Detection of gene expression with GeneChip microarray}

Total RNA was extracted from the FeNP-treated and untreated control cells with Trizol reagent. The total RNA was quantified using a UV spectrophotometer (NanoDrop 1000) and stored at $-80^{\circ} \mathrm{C}$ for later use. To profile the global gene expression, the RNA samples were analyzed using Affymetrix Human Genome U133 Plus 2.0 GeneChips ${ }^{\circ}$ microarrays according to standard Affymetrix protocols. Briefly, biotin-labeled cDNA was generated from the RNA using an in vitro transcription reaction in the presence of biotin-labeled ribonucleotides. The labeled cDNA was fragmented and hybridized with the Affymetrix Human Genome U133 Plus 2.0 GeneChips $s^{\circ}$ microarrays at $45^{\circ} \mathrm{C}$ for $16 \mathrm{~h}$ in an Affymetrix hybridization oven. After hybridization, the arrays were washed, stained with streptavidin-phycoerythrin, and scanned with the Affymetrix GeneChip Scanner3000 7G. Image quantitation was performed using GeneChip ${ }^{\circ}$ Operating Software.

\section{Data analysis of the GeneChip microarray}

Following normalization and background filtration, the signal intensity data of the treated cells were compared with those of the control cells. The transcripts with intensity ratios $\geq 2$ or $\leq-2$ were identified as DEGs [70]. The functional annotation analysis was performed by uploading on the DEGs to DAVID (v6.7 [71]). The enriched GO functions including the biological processes, cellular components and molecular function with $\mathrm{p}$ values less than 0.05 were considered significant. The enriched KEGG pathways were determined with DAVID.

\section{Detection of gene expression with qPCR}

One $\mu \mathrm{g}$ of total RNA was reverse transcribed into cDNA by using the Reverse Transcriptase Kit. The cDNA was used as template to quantify the transcription of genes with quantitative PCR on a StepOne Plus instrument (Applied Biosystem) using Fast SYBR Master Mix. The primers were as follows ( $5^{\prime}$ to $\left.3^{\prime}\right)$ : Adamdec1: AGA 
CTG TGA TTG TGG CTC TCC T, TTG TCC TGG CAA GGT AGC ATC T; Ebi3: GCT CAG GAC CTC ACA GAC TAC G, GCA GCA GCA AAG CAA GGA CTC; $C y b b$ : AGG GTC AAG AAC AGG CTA AGG A, AGC AGG ACT AGA TGA GCC AGA G; Rps11: ACA TTC AGA CTG AGC GTG CC, GGA GCT TCT CCT TGC CAG TT; Id3: CAC CTT CCC ATC CAG ACA GCC, GCT TCC GGC AGG AGA GGT TC; Etv5: CCT GAG AGA CTG GAA GGC AAA, TCA TAG TTC ATG GCT GGC CG; Tmem40: ACC GTA TCC ACA GCG TCC TC, ATT GGT CTG GCT TGG TCT CCT; Hpd: GCC TCT AGT CCC AGT AGG AG, TCG CCC ATC TCT TTG TTC CA; Ifi27: TCC TTC TTT GGG TCT GGC TGA A, CAT GGG CAC AGC CAC AAC TC; Gapdh: ATT TGG TCG TAT TGG GCG, CTC GCT CCT GGA AGA TGG. A melting curve analysis was performed at the end of the PCR programs to confirm the specificity of PCR amplification. The relative quantification (RQ) was calculated using the $2^{-\Delta \Delta C t}$ method.

\begin{abstract}
Abbreviations
FeNP: Iron nanoparticle; DEG: Differentially expressed gene; TEM: Transmission electron microscopy; DMEM: Dulbecco's modified Eagle medium; DMSA: m-2,3-dimercaptosuccinic acid; qPCR: Quantitative PCR; ROS: Reactive oxygen species; CNS: Central nervous system; IdFeNPs: Low-dose FeNPs; hdFeNPs: High-dose FeNPs; MMP: Matrix metalloproteinase; FeRGs: FeNPresponsive genes; DAVID: Database for Annotation, Visualization and Integrated Discovery; GO: Gene ontology; KEGG: Kyoto Encyclopedia of Genes and Genomes; HEPES: 4-(2-Hydroxyethyl)-1-piperazineethanesulfonic acid.
\end{abstract}

\section{Competing interests}

The authors declare that they have no competing interests.

\section{Authors' contributions}

$L Z, X W, J Z$ and YL performed the experiments; LZ analyzed data; LZ and JW wrote the manuscript. All authors read and approved the final manuscript.

\section{Acknowledgements}

This work was partially supported by the grants from the National Important Science Research Program of China (2011CB933503, 2006CB933205), the National Natural Science Foundation of China (61171030), and the Technology Support Program of Jiangsu (BE2012741).

\section{Author details}

'State Key Laboratory of Bioelectronics, Southeast University, Nanjing 210096, China. ${ }^{2}$ School of Biomedical Engineering, Hubei University of Science and Technology, Xianning 437000, China.

Received: 5 September 2014 Accepted: 30 December 2014

Published online: 17 January 2015

\section{References}

1. Sun C, Lee JS, Zhang M. Magnetic nanoparticles in MR imaging and drug delivery. Adv Drug Deliv Rev. 2008;60:1252-65.

2. Salata O. Applications of nanoparticles in biology and medicine. J Nanobiotechnol. 2004;2:3.

3. Karlsson HL, Cronholm P, Gustafsson J, Moller L. Copper oxide nanoparticles are highly toxic: a comparison between metal oxide nanoparticles and carbon nanotubes. Chem Res Toxicol. 2008;21:1726-32.

4. Soenen SJ, De Cuyper M. Assessing iron oxide nanoparticle toxicity in vitro: current status and future prospects. Nanomedicine (Lond). 2010;5:1261-75.

5. Singh N, Jenkins GJ, Asadi R, Doak SH. Potential toxicity of superparamagnetic iron oxide nanoparticles (SPION). Nano Rev. 2010;1:5358.
6. Brayner R. The toxicological impact of nanoparticles. Nano Today. 2008;3:48-55.

7. Liu Y, Chen Z, Wang J. Systematic evaluation of biocompatibility of magnetic $\mathrm{Fe}_{3} \mathrm{O}_{4}$ nanoparticles with six different mammalian cell lines. J Nanopart Res. 2010;13:199-212.

8. Yang FY, Yu MX, Zhou Q, Chen WL, Gao P, Huang Z. Effects of iron oxide nanoparticle labeling on human endothelial cells. Cell Transplant. 2012;21:1805-20.

9. Liu Y, Chen Z, Gu N, Wang J. Effects of DMSA-coated $\mathrm{Fe}_{3} \mathrm{O}_{4}$ magnetic nanoparticles on global gene expression of mouse macrophage RAW264.7 cells. Toxicol Lett. 2011;205:130-9.

10. Liu Y, Zou J, Wang X, Wang T, Wang J. Effects of 2,3-Dimercaptosuccinic Acid-Coated $\mathrm{Fe}_{3} \mathrm{O}_{4}$ Nanoparticles on Genes in Two Mouse Cell Lines. J Biomed Nanotechnol. 2014;10:1574-87.

11. Khan MI, Mohammad A, Patil G, Naqvi SA, Chauhan LK, Ahmad I. Induction of ROS, mitochondrial damage and autophagy in lung epithelial cancer cells by iron oxide nanoparticles. Biomaterials. 2012;33:1477-88.

12. Baratli Y, Charles AL, Wolff V, Ben Tahar L, Smiri L, Bouitbir J, et al. Age modulates $\mathrm{Fe}_{3} \mathrm{O}_{4}$ nanoparticles liver toxicity: dose-dependent decrease in mitochondrial respiratory chain complexes activities and coupling in middle-aged as compared to young rats. Biomed Res Int. 2014:2014:474081

13. Liu Y, Huang J, Wang D, Wang J. Identification of DMSA-coated $\mathrm{Fe}_{3} \mathrm{O}_{4}$ nanoparticles induced-apoptosis response genes in human monocytes by cDNA microarrays. Adv Mater Res. 2013;749:377-83.

14. Zhu MT, Wang $Y$, Feng $W Y$, Wang $B$, Wang $M$, Ouyang $H$, et al. Oxidative stress and apoptosis induced by iron oxide nanoparticles in cultured human umbilical endothelial cells. J Nanosci Nanotechnol. 2010;10:8584-90.

15. Diana V, Bossolasco P, Moscatelli D, Silani V, Cova L. Dose dependent side effect of superparamagnetic iron oxide nanoparticle labeling on cell motility in two fetal stem cell populations. PLoS One. 2013;8:e78435.

16. Kedziorek DA, Muja N, Walczak P, Ruiz-Cabello J, Gilad AA, Jie CC, et al. Gene expression profiling reveals early cellular responses to intracellular magnetic labeling with superparamagnetic iron oxide nanoparticles. Magn Reson Med. 2010;63:1031-43.

17. Alarifi S, Ali D, Alkahtani S, Alhader MS. Iron oxide nanoparticles induce oxidative stress, DNA damage, and caspase activation in the human breast cancer cell line. Biol Trace Elem Res. 2014;159:416-24.

18. Ahamed M, Alhadlaq HA, Alam J, Khan MA, Ali D, Alarafi S. Iron oxide nanoparticle-induced oxidative stress and genotoxicity in human skin epithelial and lung epithelial cell lines. Curr Pharm Des. 2013;19:6681-90.

19. Li X, He Q, Shi J. Global gene expression analysis of cellular death mechanisms induced by mesoporous silica nanoparticle-based drug delivery system. ACS Nano. 2014;8:1309-20.

20. Foldbjerg R, Irving ES, Hayashi Y, Sutherland DS, Thorsen K, Autrup H, et al. Global gene expression profiling of human lung epithelial cells after exposure to nanosilver. Toxicol Sci. 2012;130:145-57.

21. Liu Y, Wang J. Effects of DMSA-coated $\mathrm{Fe}_{3} \mathrm{O}_{4}$ nanoparticles on the transcription of genes related to iron and osmosis homeostasis. Toxicol Sci. 2013;131:521-36

22. van Aerle R, Lange A, Moorhouse A, Paszkiewicz K, Ball K, Johnston BD, et al. Molecular mechanisms of toxicity of silver nanoparticles in zebrafish embryos. Environ Sci Technol. 2013;47:8005-14.

23. Waters KM, Masiello LM, Zangar RC, Tarasevich BJ, Karin NJ, Quesenberry RD, et al. Macrophage responses to silica nanoparticles are highly conserved across particle sizes. Toxicol Sci. 2009;107:553-69.

24. Simon DF, Domingos RF, Hauser C, Hutchins CM, Zerges W, Wilkinson KJ. Transcriptome sequencing (RNA-seq) analysis of the effects of metal nanoparticle exposure on the transcriptome of Chlamydomonas reinhardtii. Appl Environ Microbiol. 2013;79:4774-85.

25. Shim W, Paik MJ, Nguyen DT, Lee JK, Lee Y, Kim JH, et al. Analysis of changes in gene expression and metabolic profiles induced by silica-coated magnetic nanoparticles. ACS Nano. 2012;6:7665-80.

26. Tiwari DK, Jin T, Behari J. Dose-dependent in-vivo toxicity assessment of silver nanoparticle in Wistar rats. Toxicol Mech Methods. 2011;21:13-24.

27. Kowarik MC, Cepok S, Sellner J, Grummel V, Weber MS, Korn T, et al. CXCL13 is the major determinant for $\mathrm{B}$ cell recruitment to the CSF during neuroinflammation. J Neuroinflammation. 2012;9:93.

28. Yamamori $H$, Hashimoto R, Ishima T, Kishi F, Yasuda Y, Ohi K, et al. Plasma levels of mature brain-derived neurotrophic factor (BDNF) and matrix metalloproteinase-9 (MMP-9) in treatment-resistant schizophrenia treated with clozapine. Neurosci Lett. 2013;556:37-41. 
29. Hsiao CP, Araneta M, Wang XM, Saligan LN. The association of IFI27 expression and fatigue intensification during localized radiation therapy: implication of a para-inflammatory bystander response. Int J Mol Sci. 2013;14:16943-57.

30. Koldehoff M, Cierna B, Steckel NK, Beelen DW, Elmaagacli AH. Maternal molecular features and gene profiling of monocytes during first trimester pregnancy. J Reprod Immunol. 2013;99:62-8.

31. Rosebeck S, Leaman DW. Mitochondrial localization and pro-apoptotic effects of the interferon-inducible protein ISG12a. Apoptosis. 2008;13:562-72.

32. Dos Santos Hidalgo G, Meola J, Rosa ESJC, Paro de Paz CC, Ferriani RA TAGLN expression is deregulated in endometriosis and may be involved in cell invasion, migration, and differentiation. Fertil Steril. 2011;96:700-3.

33. Liu $\mathrm{H}$. The immunotherapeutic effect of $\mathrm{Fe}_{3} \mathrm{O}_{4}$ nanoparticles as adjuvants on mice He22 live cancer. J Nanosci Nanotechnol. 2010;10:514-9.

34. Lauper N, Beck AR, Cariou S, Richman L, Hofmann K, Reith W, et al. Cyclin E2: a novel CDK2 partner in the late G1 and S phases of the mammalian cell cycle. Oncogene. 1998;17:2637-43.

35. Gelman IH. Emerging roles for SSeCKS/Gravin/AKAP12 in the control of cell proliferation, cancer malignancy, and barriergenesis. Genes Cancer. 2010;1:1147-56.

36. Gelman IH. Suppression of tumor and metastasis progression through the scaffolding functions of SSeCKS/Gravin/AKAP12. Cancer Metastasis Rev. 2012;31:493-500.

37. Fukuda N. Cigarette smoking induces vascular proliferative disease through the activation of Egr-1. Cardiovasc Res. 2010;88:207-8.

38. Mayer SI, Rossler OG, Endo T, Charnay P, Thiel G. Epidermal-growth-factorinduced proliferation of astrocytes requires Egr transcription factors. J Cell Sci. 2009;122:3340-50.

39. Felty $\mathrm{Q}$, Porther N. Estrogen-induced redox sensitive Id 3 signaling controls the growth of vascular cells. Atherosclerosis. 2008;198:12-21

40. Nickenig G, Baudler S, Muller C, Werner C, Werner N, Welzel H, et al. Redoxsensitive vascular smooth muscle cell proliferation is mediated by GKLF and Id3 in vitro and in vivo. FASEB J. 2002;16:1077-86.

41. Takeda K, Akira S. Toll-like receptors in innate immunity. Internat Immunol. 2005;17:1-14.

42. Liu YX, Chen ZP, Wang JK. Internalization of DMSA-coated $\mathrm{Fe}_{3} \mathrm{O}_{4}$ magnetic nanoparticles into mouse macrophage cells. Adv Mater Res. 2012:455-456:1221-7.

43. Wong MM, Fish EN. Chemokines: attractive mediators of the immune response. Semin Immunol. 2003;15:5-14

44. Nibbs RJB, Graham GJ. Immune regulation by atypical chemokine receptors. Nat Rev Immunol. 2013;13:815-29.

45. Lee YS, Dutta A. MicroRNAs in cancer. Annu Rev Pathol. 2009:4:199-227.

46. Babashah S, Soleimani M. The oncogenic and tumour suppressive roles of microRNAs in cancer and apoptosis. Eur J Cancer. 2011;47:1127-37.

47. Chung RT, Gale Jr M, Polyak SJ, Lemon SM, Liang TJ, Hoofnagle JH. Mechanisms of action of interferon and ribavirin in chronic hepatitis $C$ summary of a workshop. Hepatology. 2008;47:306-20.

48. Mourskaia AA, Northey JJ, Siegel PM. Targeting aberrant TGF-beta signaling in pre-clinical models of cancer. Anticancer Agents Med Chem. 2007;7:504-14

49. Kang YJ, Shin JW, Yoon JH, Oh IH, Lee SP, Kim SY, et al. Inhibition of erythropoiesis by Smad6 in human cord blood hematopoietic stem cells. Biochem Biophys Res Commun. 2012:423:750-6.

50. Guo Q, Guo P, Mao Q, Lan J, Lin Y, Jiang J, et al. ID1 affects the efficacy of radiotherapy in glioblastoma through inhibition of DNA repair pathways. Med Oncol. 2013;30:325

51. Norton JD. ID helix-loop-helix proteins in cell growth, differentiation and tumorigenesis. J Cell Sci. 2000;113(Pt 22):3897-905.

52. Sikder HA, Devlin MK, Dunlap S, Ryu B, Alani RM. Id proteins in cell growth and tumorigenesis. Cancer Cell. 2003;3:525-30.

53. Petit V, Thiery JP. Focal adhesions: structure and dynamics. Biol Cell. 2000:92:477-94

54. Raynal I, Prigent P, Peyramaure S, Najid A, Rebuzzi C, Corot C. Macrophage endocytosis of superparamagnetic iron oxide nanoparticles: mechanisms and comparison of ferumoxides and ferumoxtran-10. Invest Radiol. 2004:39:56-63.

55. Lunov O, Zablotskii V, Syrovets T, Rocker C, Tron K, Nienhaus GU, et al. Modeling receptor-mediated endocytosis of polymer-functionalized iron oxide nanoparticles by human macrophages. Biomaterials. 2011;32:547-55.

56. Canete M, Soriano J, Villanueva A, Roca AG, Veintemillas S, Serna CJ, et al. The endocytic penetration mechanism of iron oxide magnetic nanoparticles with positively charged cover: a morphological approach. Int J Mol Med 2010;26:533-9.

57. Osman O, Zanini LF, Frenea-Robin M, Dumas-Bouchiat F, Dempsey NM, Reyne $\mathrm{G}$, et al. Monitoring the endocytosis of magnetic nanoparticles by cells using permanent micro-flux sources. Biomed Microdevices. 2012;14:947-54

58. Mueller C, Baudler S, Welzel H, Bohm M, Nickenig G. Identification of a novel redox-sensitive gene, Id3, which mediates angiotensin II-induced cell growth. Circulation. 2002;105:2423-8.

59. Singh N, Jenkins GJ, Nelson BC, Marquis BJ, Maffeis TG, Brown AP, et al. The role of iron redox state in the genotoxicity of ultrafine superparamagnetic iron oxide nanoparticles. Biomaterials. 2012;33:163-70.

60. Auffan M, Achoulak W, Rose J, Roncato MA, Chaneac C, Waite DT, et al. Relation between the redox state of iron-based nanoparticles and their cytotoxicity toward Escherichia coli. Environ Sci Technol. 2008;42:6730-5.

61. Murray AR, Kisin E, Inman A, Young SH, Muhammed M, Burks T, et al. Oxidative stress and dermal toxicity of iron oxide nanoparticles in vitro. Cell Biochem Biophys. 2013;67:461-76.

62. Keenan CR, Goth-Goldstein R, Lucas D, Sedlak DL. Oxidative stress induced by zero-valent iron nanoparticles and Fe(II) in human bronchial epithelial cells. Environ Sci Technol. 2009:43:4555-60.

63. Sevcu A, El-Temsah YS, Joner EJ, Cernik M. Oxidative stress induced in microorganisms by zero-valent iron nanoparticles. Microbes Environ. 2011;26:271-81.

64. Simbulan-Rosenthal CM, Daher A, Trabosh V, Chen WC, Gerstel D, Soeda E, et al. Id3 induces a caspase-3- and -9-dependent apoptosis and mediates UVB sensitization of HPV16 E6/7 immortalized human keratinocytes. Oncogene. 2006;25:3649-60.

65. Kee BL. Id3 induces growth arrest and caspase-2-dependent apoptosis in B lymphocyte progenitors. J Immunol. 2005;175:4518-27.

66. Wilson JW, Deed RW, Inoue T, Balzi M, Becciolini A, Faraoni P, et al. Expression of Id helix-loop-helix proteins in colorectal adenocarcinoma correlates with p53 expression and mitotic index. Cancer Res. 2001;61:8803-10.

67. Yang HY, Liu HL, Ke J, Wu H, Zhu H, Liu JR, et al. Expression and prognostic value of Id protein family in human breast carcinoma. Oncol Rep. 2010;23:321-8.

68. Castanon E, Bosch-Barrera J, Lopez I, Collado V, Moreno M, Lopez-Picazo JM et al. Id1 and Id3 co-expression correlates with clinical outcome in stage III-N2 non-small cell lung cancer patients treated with definitive chemoradiotherapy. J Transl Med. 2013;11:13.

69. Chen ZP, Zhang Y, Zhang S, Xia JG, Liu JW, Xu K, et al. Preparation and characterization of water-soluble monodisperse magnetic iron oxide nanoparticles via surface double-exchange with DMSA. Colloid Surf A Physicochem Eng Asp. 2008;316:210-6.

70. Vasil'eva LL, Kochan VA, Obelevskaya KM. Extending the field of application of IPS-020 and IPS-101 power supplies. Meas Tech. 1972;15:1719.

71. Dennis Jr G, Sherman BT, Hosack DA, Yang J, Gao W, Lane HC, et al. DAVID: Database for Annotation, Visualization, and Integrated Discovery. Genome Biol. 2003;4:P3.

\section{Submit your next manuscript to BioMed Central and take full advantage of:}

- Convenient online submission

- Thorough peer review

- No space constraints or color figure charges

- Immediate publication on acceptance

- Inclusion in PubMed, CAS, Scopus and Google Scholar

- Research which is freely available for redistribution 